\title{
Linear and Hyperpolarizabilities of Spiropyran and Derivatives
}

\author{
M.K. SABRA* \\ Physics Department, Atomic Energy Commission of Syria \\ P.O. Box 6091, Damascus, Syria \\ (Received June 19, 2008; in final form September 29, 2008)
}

\begin{abstract}
The geometry of spiropyran molecule and its derivatives was found using quasi-Newton methods, BroydenFletcher-Goldfarb-Shanno technique. Then ab initio quantum chemical methods were applied to calculate the ground state energies of the closed and open forms of spiropyran for different basis sets. The frequency dependent linear polarizability of spiropyran and derivatives were obtained and plotted. The found absorption peaks are different for the closed and the open structures of the spiropyran molecule.
\end{abstract}

PACS numbers: 31.15.A-, 32.10.Dk, 33.15.Kr

\section{Introduction}

The interest in organic materials over the last decades did not only lie in their enhanced electric responses over a wide frequency range and ultrafast response times, but also in the many ways of varying their molecular structures [1]. From the beginning, this interest was stimulated mainly by the potential applications of organic materials in nonlinear optical devices due to their high hyperpolarizabilities. High electric susceptibility very much depends on the nature of the delocalized network of $\pi$ electrons and it is important to find molecular structures that yield the largest possible responses because, among other conditions and advantages, the larger the nonlinear response, the smaller the electric field required to achieve the desired nonlinear effect. The molecular structure of the organic materials can easily be modified in order to maximize their electric responses, but this versatility has also its drawback because, due to cost and time, it is practically impossible to prepare and test all interesting compounds. Moreover, since it is expected that the compounds used to form materials will depend on the particular application under consideration, there will be a continuous need for designing new molecules.

Photochromic materials are of interest to a variety of potential applications including high resolution photography, optical devices [2,3], holographic systems, variable transmission devices as lenses and filters, and erasable memory media $[4,5]$. The photochromic polymers have many advantages in the manufacture of organic devices for integrated optics as the flexibility of ac-

\footnotetext{
* e-mail: mkhsabra@aec.org.sy
}

commodating different chromophores $[6,7]$ and the possibility of combining two different properties in the same matrix. The photorefractive polymers provide a good example, as they combine photoconducting and second harmonic generation properties.

Atassi et al. [8, 9] reported experimentally two new classes of materials that exhibit the two distinct properties, photochromism and second order nonlinear optical properties; spiropyran (SP)-photomerocyanine (PM) in polymer systems. Spiropyran is one type of organic photochromic molecule that upon application of ultraviolet irradiation interconverts into colored photomerocyanine form. The photomerocyanine converts back to the spiropyran form either thermally or photochemically. It also has two structural forms quinonic and zwitterionic. The zwitterionic photomerocyanine form has extended planer electronic conjugation that causes the optical shift. Figure 1 shows the reversible photochemical reaction of the spiropyran-photomerocyanine system [10] and the formulae of the different derivatives.

Beside the above experimental work there was a little theoretical work to calculate the first order hyperpolarizability of SP by finite field methods in combination with the modified neglect of differential overlap (MNDO) molecular orbital method [8]. The frequency-dependent hyperpolarizabilities of SP and derivatives have not been reported yet.

The main goal of this work is to calculate frequency-dependent polarizabilities of the $\mathrm{SP}$ and derivatives in the framework of the $a b$ initio theory. Different basis sets have been employed for this purpose. We will not report all results for all the used basis sets, but we will quote them as needed. 


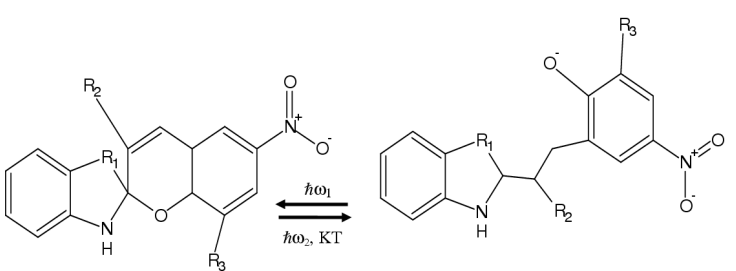

\begin{tabular}{|l|c|c|c|}
\hline \multicolumn{1}{|c|}{ Derivative } & $\mathrm{R}_{1}$ & $\mathrm{R}_{2}$ & $\mathrm{R}_{3}$ \\
\hline $\begin{array}{l}\mathrm{SP}_{1}: \text { 6-nitro-1',3',3'-trimethylspiro[2H- } \\
\text { 1-benzopyran] }\end{array}$ & $-\mathrm{C}(\mathrm{CH} 3) 2-$ & $-\mathrm{H}$ & $-\mathrm{H}$ \\
\hline $\begin{array}{l}\mathrm{SP}_{2}: \text { 3-ethoxy-8-methoxy-6-nitro-3'- } \\
\text { methylspiro[2H-1-benzopyran-2,2'- } \\
\text { benzothiazoline] }\end{array}$ & $-\mathrm{S}$ & -OC2H5 & -OCH3 \\
\hline $\begin{array}{l}\mathrm{SP}_{3}: \text { 8-methoxy-3-methyl- 6-nitro-3'- } \\
\text { methylspiro[2H-1-benzopyran-2,2'- } \\
\text { benzothiazoline] }\end{array}$ & $-\mathrm{S}$ & $-\mathrm{CH} 3$ & -OCH3 \\
\hline $\begin{array}{l}\mathrm{SP}_{4}: \text { 8-(ethylthio)methyl-6-nitro-1',3', } \\
\text { 3'- trimethylspiro [2H-1- benzopyran- } \\
\text { 2,2'-indoline] }\end{array}$ & $-\mathrm{C}(\mathrm{CH} 3) 2$ & $-\mathrm{H}$ & -CH2SCH5 \\
\hline
\end{tabular}

Fig. 1. The reversible photochemical reaction of the spiropyran-photomerocyanine system and the formulae of the different derivatives.

\section{Theoretical models}

The time dependent Hartree-Fock approach (TDHF) [11] in the framework of the $a b$ initio quantum chemical methods are implemented in the General Atomic and Molecular Electronic Structure System code (GAMESS-US) [12]. This approach permits the analytic calculation of various static and frequency dependent polarizabilities, with an emphasis on important nonlinear optical (NLO) properties such as second and third harmonic generation. The method is programmed only for closed shell wave functions, at the semi-empirical or ab initio level. The restricted Hartree-Fock (RHF) approximation in the framework of the Austin model (AM1) and MNDO basis sets have been used to calculate the frequency-dependent linear and nonlinear polarizabilities of the SP and its derivatives. In addition the Slater type orbital (STO) basis set has been used to study the effect of the basis sets on the polarizabilities.

Polarizabilities are sensitive to the dipole moment, which is connected directly to the geometry of the molecule. Delaire et al. [9] reported two different values of the first hyperpolarizability $\beta$, for example, of PM1 starting from the crystallographic data and further optimization of angles and bond lengths. In real systems molecules should be in the ground states of energies with respect to geometrical structure. Therefore all molecules under consideration, closed and open forms, are subjected to geometrical optimization using the quasi-Newton methods, Broyden-Fletcher-Goldfarb-Shanno (BFGS) technique [13].

\section{Results and discussion}

A specially written program based on the BFGS technique has been used to optimize all the molecules under investigation starting from a trial structure. The output Cartesian coordinates have been fed as input to the
GAMESS-US program to calculate the ground state energies, linear and nonlinear polarizabilities. All molecules structures under consideration are non centro-symmetric, so odd and even polarizabilities can exist.

The ground states energies of spiropyran and its derivatives are recorded in Tables I and II, The ground states energies and diploe moments of spiropyran and its derivatives are recorded in table I and II using RHF approximation in the framework of STO-4G basis set, respectively - Whereas all molecules in the closed form are lower in energy and under UV illumination, they undergo a ring opening converting into metastable states, the open forms. These metastable states go back through photochemical or thermal reactions.

\section{TABLE I}

The ground state energies (Hartree) of spiropyran and its derivatives for STO-4G basis sets.

\begin{tabular}{c|c|c|c}
\hline \hline Molecule & Closed & Quinonic & Zwitterionic \\
\hline SP1 & -1018.6841 & -1018.6506 & -1018.3858 \\
SP2 & -1562.9525 & -1562.4884 & -1562.1859 \\
SP3 & -1449.7348 & -1449.0380 & -1448.9787 \\
SP4 & -1530.6972 & -1529.9528 & -1529.7342
\end{tabular}

TABLE II

The dipole moment (Debye) of spiropyran and its derivatives for STO-4G basis sets.

\begin{tabular}{c|c|c|c}
\hline \hline Molecule & Closed & Quinonic & Zwitterionic \\
\hline SP1 & 4.27 & 6.54 & 17.16 \\
SP2 & 5.73 & 5.54 & 16.21 \\
SP3 & 5.54 & 5.52 & 13.86 \\
SP4 & 4.46 & 6.40 & 14.02
\end{tabular}

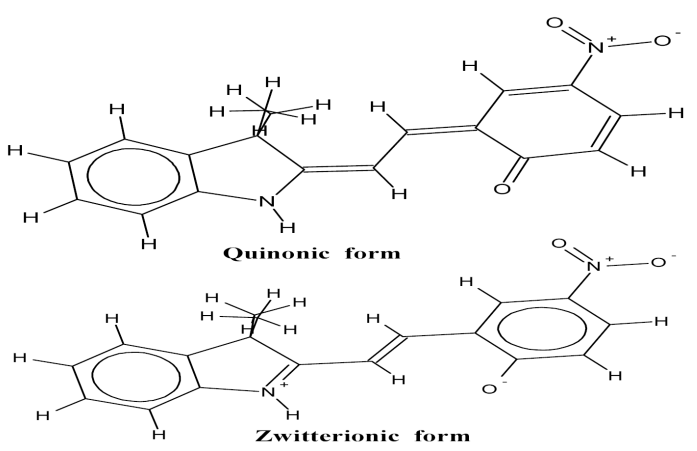

Fig. 2. The mesomeric forms of photomerocyanine in case of PM1.

Some of these open forms have two different structures, the quinonic and the zwitterionic forms of photomerocyanine (see Fig. 2). The only difference between these 
two forms is the bond length alternation (BLA) order, the segment that connects the $\pi$ rings. The BLA order is defined as the average differences in length between adjacent single and double bonds along the conjugated segment [14]. For the open forms of spiropyran, BLA order plays an important parameter to determine the value of the hyperpolarizabilities. As an example, we illustrate the results of SP1 in detail in the next section and then the results of SP2, SP3 and SP4.

$S P 1$. Before proceeding in reporting the polarizabilities, we would like to have a look at the effect of the BLA on the ground state of PM1 in both cases of quinonic and zwitterionic structures.

Bond length alternation order. After optimizing the quinonic and zwitterionic structures of PM1 as stated above using the BGFS technique, there are many ways to change the BLA in the 3D space. We are not interested to span all the possible ways, but what has been done is to displace the carbon atoms from their obtained optimized positions, manually.

For each BLA value, the total energy and dipole moment have been calculated in the framework of AM1 basis set for both forms, to compare our results with others. Figure 3 represents the total energies of the quinonic and the zwitterionic structures of PM1 as a function of BLA, where the quinonic form is lower in energy for the whole range of BLA. In addition, they behave parabolic in terms of BLA, indicating that the displacements are linear around the equilibrium positions.

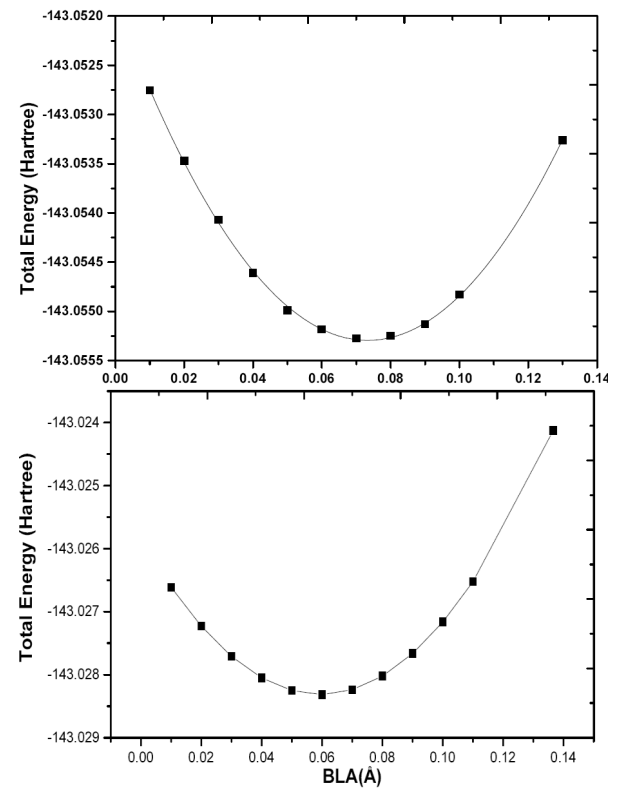

Fig. 3. The total energy of the quinonic (top) and the zwitterionic (bottom) forms of photomerocyanine PM1 in terms of BLA.

Fitting each plot to a quadratic equation in BLA, we find the spring constant of the bonds to be $34.6 \mathrm{eV} \AA^{-2}$ and $\mathrm{BLA}=0.073 \AA$ corresponding to the minimum en- ergy in case of the quinonic structure in the gas phase. This BLA value is larger than that found by Delaire et al. [9], $0.035 \AA$ through further optimization of the 3D crystallographic data using the MOPAC molecular orbital program, while in the zwitterionic case, the spring constant is $38.2 \mathrm{eV} \AA^{-2}$ and the BLA that corresponds to the minimum energy is $-0.059 \AA$. This value is smaller than that found in the $3 \mathrm{D}$ crystallographic data [9], $-0.086 \AA$.

After optimizing the geometries of the molecules under consideration, the GAMESS input file has been modified to calculate the linear and nonlinear polarizabilities for different frequencies in the framework of MNDO basis set. The range of frequencies have been chosen from zero, to calculate the static polarizabilities, up to $0.1 \mathrm{Ha}$ i.e. it covers a range from the spectrum from far infrared up to violet.

The nonlinear optical response of an isolated molecule in an electric field $E_{i}(\omega)$ is given by many authors in the literature. As the values of the polarizabilities that obtained theoretically by different methods may be different, it seems necessary to give an exact definition. The calculated isotropic average $\alpha(\omega)$ in this work is given as

$$
\alpha(\omega)=\frac{1}{3} \sum_{i=x, y, z} \alpha_{i i}(\omega),
$$

where $\alpha_{i}(\omega)$ is the polarizability in the $i$-th direction.

$\alpha(\omega)$ of the closed, the quinonic and the zwitterionic forms of SP1 in terms of frequency have been plotted in Fig. 4. Whereas $\alpha(\omega)$ of the three forms increase monotonically, while it increases more rapidly for the zwitterionic form in the region $2-2.5 \mathrm{eV}$. The polarizability is given in terms of the frequency as [15]:

$$
\alpha(\omega) \sim\left(w^{2}-w_{0}^{2}\right)^{-1} .
$$

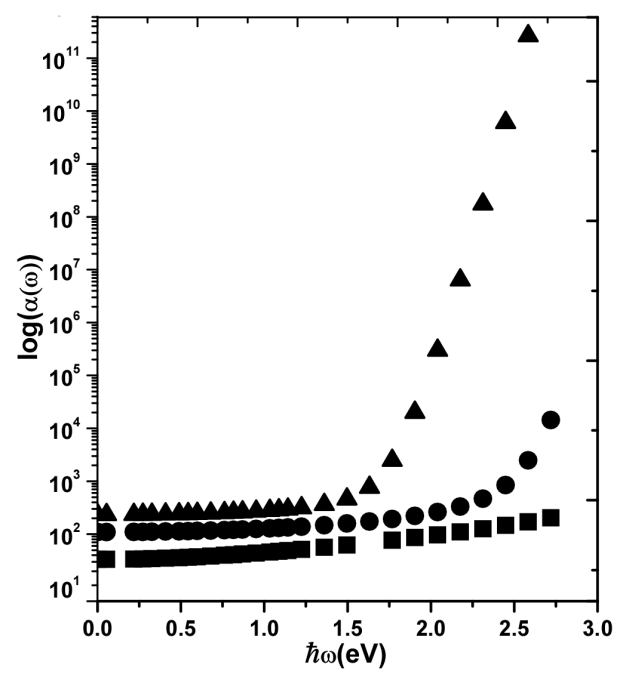

Fig. 4. The linear polarizabilities of the closed (-), the quinonic $(\bullet)$ and the zwitterionic $(\boldsymbol{\Delta})$ forms of SP1 in terms of the frequency. 
Fitting each plot with Eq. (2), the maxima are found to occur at wavelengths $346 \mathrm{~nm}, 370 \mathrm{~nm}$, and $485 \mathrm{~nm}$ for the closed, the quinonic and the zwitterionic forms, respectively. It is clear that the peak of the absorption of the closed form is around $350 \mathrm{~nm}$, which is in good agreement with the experiment [8]. And there is a shift from ultraviolet to violet and to blue. The blue color of the zwitterionic photomerocyanine has been confirmed by Hirano et al. [16, 17].

With analogy to the linear polarizability, the theoretically calculated second harmonic polarizability may differ with the used method. Thus, we define $\beta$ as

$$
\beta_{k}=\sum_{i=x, y, z} \beta_{k i i}+\beta_{i k i}+\beta_{i i k}, \quad k=x, y, z
$$

and

$$
\beta=\sqrt{\beta_{x}^{2}+\beta_{y}^{2}+\beta_{z}^{2}} .
$$

The frequency-dependent $\beta(2 \omega)$ of the closed, the quinonic and the zwitterionic forms of SP1 have been plotted in Fig. 5 as functions of the frequency in the range 0-2.0 eV. The three functions increase rapidly with the frequency, especially in the range $1.0-2.1 \mathrm{eV}$. The frequency response of $\beta$ for the frequency doubling process can be written according to the two-level model approximation [18] as

$$
\beta \propto \frac{A}{\left(\omega_{0}^{2}-\omega^{2}\right)\left(\omega_{0}^{2}-4 \omega^{2}\right)},
$$

where $A$ is a physical constant and $\omega_{0}$ is the difference between excited- and ground-state dipole moments. Fitting each function with Eq. (5) we obtain the resonance frequency for the closed, the quinonic and the zwitterionic forms occur at $651,754,1000 \mathrm{~nm}$, respectively. These frequencies are not exactly double those frequencies found before for $\alpha(\omega)$. Such discrepancy can be referred to approximated model of two levels and to numerical calculations.

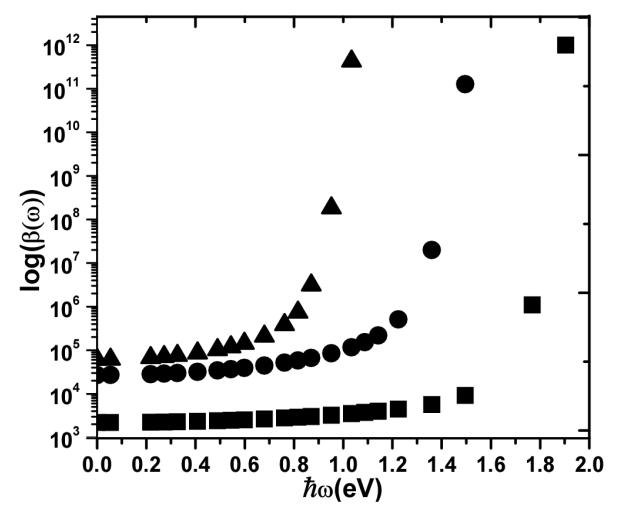

Fig. 5. The second harmonic polarizabilities of the closed $(\boldsymbol{\bullet})$, the quinonic $(\bullet)$ and the zwitterionic $(\boldsymbol{\Delta})$ forms of SP1 in terms of the frequency.

Again here, the theoretically calculated $\gamma(3 \omega)$ may differ with the used method. Therefore, it will be clearer if we define our calculated third harmonic generation po- larizability as

$$
\gamma=\frac{1}{5} \sum_{i=x, y, z} \gamma_{i i x x}+\gamma_{i i y y}+\gamma_{i i z z} .
$$

Figure 6 shows the $\gamma(3 \omega)$ of the closed, the quinonic and the zwitterionic structures of SP1 as stated in Eq. (6). For all forms, $\gamma(3 \omega)$ increases monotonically with the frequency. The general form of $\gamma(3 \omega)$ in terms of the frequency is given by many authors in the literature. In the purpose of finding the peaks of the absorption, we think it is enough to fit our data to one term of the polarizability formula as

$$
\gamma(3 \omega) \sim \frac{A}{\left(\omega-\omega_{1}\right)\left(\omega-\omega_{2}\right)\left(\omega-\omega_{3}\right)},
$$

where this term have three singularities. Fitting each curve to Eq. (7), the peaks of the closed, the quinonic, and the zwitterionic structures would occur at 1032, 1139 and $1458 \mathrm{~nm}$, respectively. There is no experimental data, up to our knowledge, to compare it with our results.

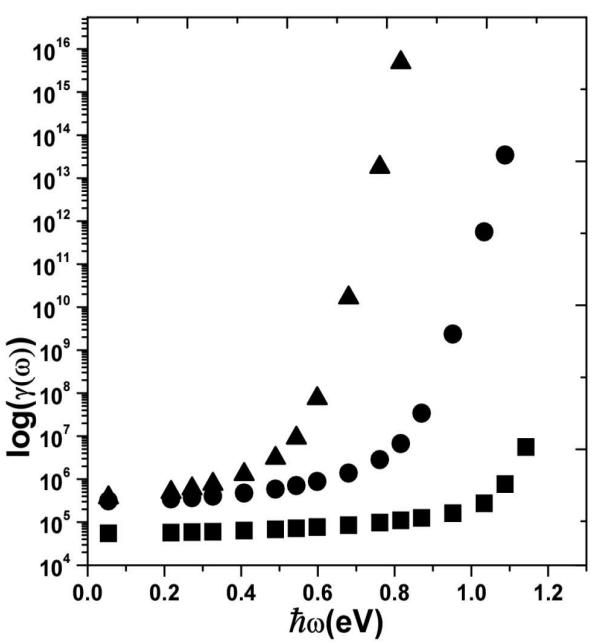

Fig. 6. The third harmonic polarizabilities of the closed $(\boldsymbol{\bullet})$, the quinonic $(\bullet)$ and the zwitterionic $(\boldsymbol{\Delta})$ forms of SP1 in terms of the frequency.

Spiropyran derivatives. As we have done in the SP1 case, after geometry optimization using BFGS method the GAMESS-US input file has been modified to calculate the frequency dependent polarizabilities, $\alpha(\omega), \beta(\omega)$ and $\gamma(\omega)$, of the closed forms of the spiropyran derivatives. Another basis set which is the STO-4G has been used to demonstrate the basis effect. The frequencies cover a part of the spectrum from far infrared up to violet.

The presented results here are restricted to the closed forms only, because we have demonstrated above an example of the behavior of the linear and nonlinear polarizabilities of the quinonic and the zwitterionic forms of SP1.

Figure 7 represent the $\alpha(\omega)$, as defined in Eq. (2), of SP2, SP3 and SP4 molecules, where all increase mono- 
tonically with frequency. Fitting each curve with Eq. (2), we find all the closed forms of the molecules under consideration have an absorption peak at 337, 310, 302 and $278 \mathrm{~nm}$ for SP1, SP2, SP3, and SP4, respectively.

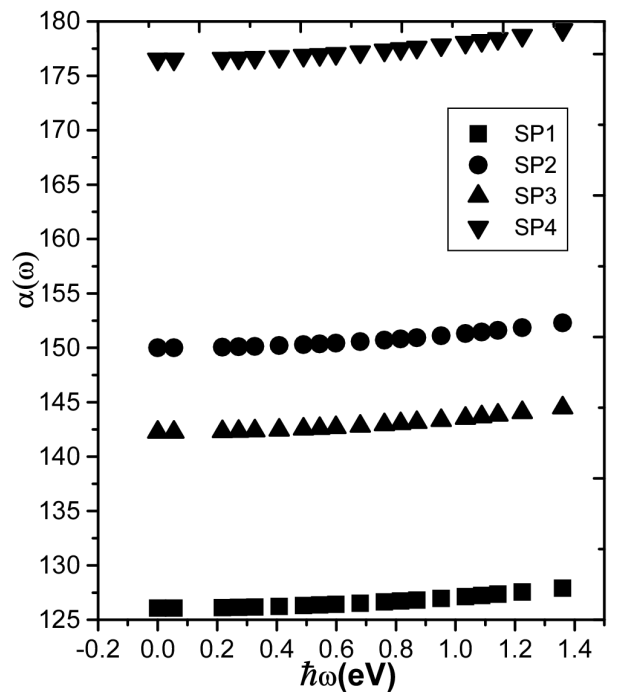

Fig. 7. The linear polarizabilities of the closed forms of the SP1 $(\boldsymbol{\bullet})$, the SP2 $(\bullet)$, the SP3 $(\boldsymbol{\Delta})$ and the SP4 $(\boldsymbol{\nabla})$ in terms of the frequency.

Figure 8 shows the first hyperpolarizability $\beta(2 \omega)$ of the four molecules calculated according to Eqs. (3), (4). Having in mind the two-level model and fitting each curve to Eq. (5), one finds that all peaks occur in the yellow region at the wavelengths about $590 \mathrm{~nm}$ for SP1, SP2, SP3 and at $578 \mathrm{~nm}$ for SP4, respectively. The result of the zwitterionic forms of SP1 is far from the experimental data.

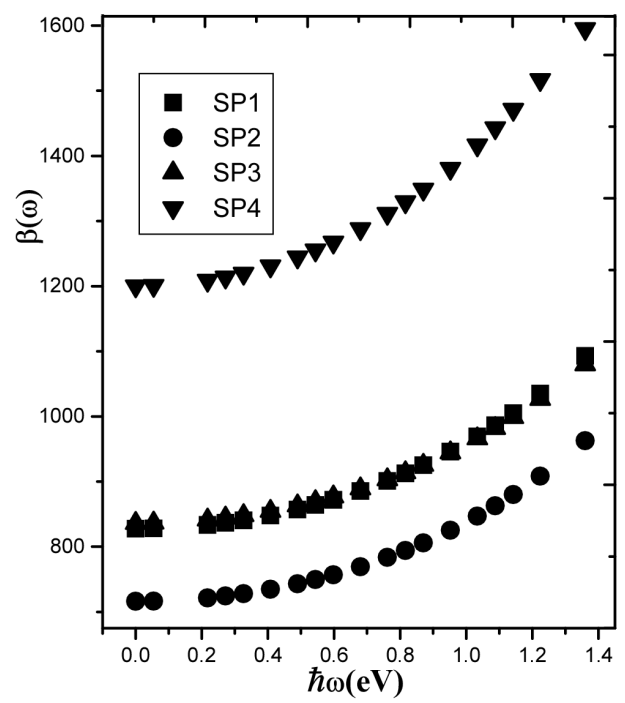

Fig. 8. The first hyperpolarizability of the closed forms of the SP1 (घ), the SP2 $(\bullet)$, the SP3 ( $\boldsymbol{\Delta})$ and the SP4 $(\boldsymbol{\nabla})$ in terms of the frequency.

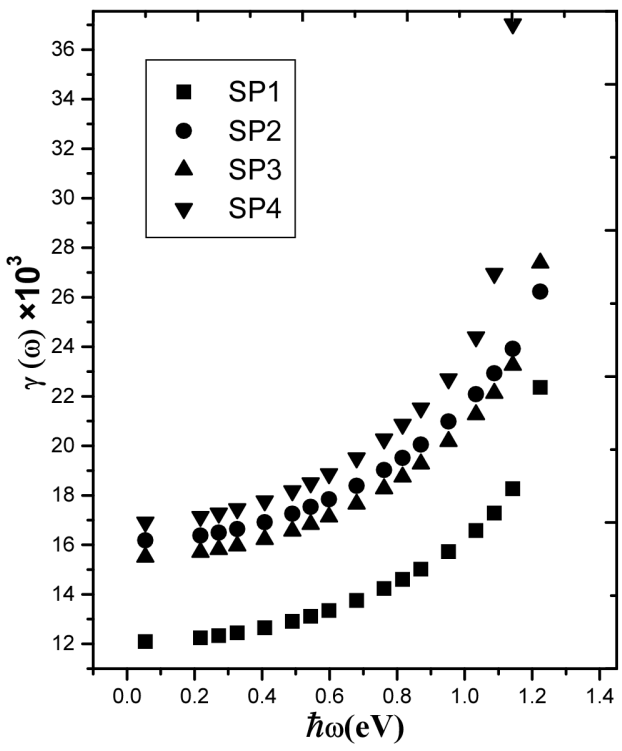

Fig. 9. The third harmonic generation polarizability of

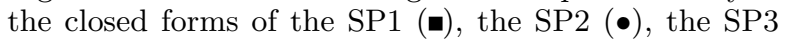
$(\boldsymbol{\Delta})$ and the SP4 $(\boldsymbol{\nabla})$ in terms of the frequency.

The third harmonic generation polarizability $\gamma(\omega)$ of the closed structures of the SP's molecules has been plotted in Fig. 9. It increases rapidly with the frequency, where someone would notice that the peaks will appear in the range of 1.0-1.2 eV corresponding to wavelength range of $1032-1240 \mathrm{~nm}$.

\section{Conclusion}

The $a b$ initio quantum chemical methods in the framework of the Hartree-Fock approximation using the Slater type orbital MNDO and STO-4G basis set have been used to calculate the linear and nonlinear polarizabilities of the spiropyran and derivatives. These polarizabilities are crucial to the geometries of the molecules. Therefore, the geometries optimization have been done using the Broyden-Fletcher-Goldfarb-Shanno technique. The ground states energy of the closed and the open structures of the spiropyran (SP1), as an example, have been calculated. It is found that the ground states energy of the open forms lower than the closed one, which is in good agreement with experiment. In addition, the peak of the linear absorption of the zwitterionic form shifts towards the blue region of the spectrum in agreement with the experimental data. The peaks of the second harmonic generation polarizabilities of the closed, the quinonic and the zwitterionic forms of SP1 are found to be deviated from the double of the $\alpha(\omega)$. This is referred to the two-level model, while the peaks of the third harmonic generation polarizabilities of SP1 are found close to the triplet of the positions of the $\alpha(\omega)$ peaks.

In addition, study of the polarizabilities of the spiropyran derivatives has been performed in the framework of the STO-4G basis. Our calculations demonstrate that 
the linear polarizability differs from one derivative to another, but all fall in the ultraviolet region, while the second harmonic generation polarizabilities peaks occur in the green spectrum. The third harmonic generation polarizabilities peaks are found to be in the infrared part of the spectrum.

\section{Acknowledgments}

The author would like to thank Prof. I. Othman for his permanent support and Mr. Y. Salman for his help.

\section{References}

[1] Nonlinear Optical Effects and Materials Series: Springer Series in Optical Sciences, Ed. P. Günter, Vol. 72, Springer, Berlin 1984.

[2] A. Bobrovsky, N. Boiko, V. Shaibaev, Adv. Mater. 11, 1025 (1999).

[3] G.R. Ramos, M.R. Delgado, C.D. Iturbe, S.E. Gonzácutelez, C.F. Soriano, Opt. Quantum Electron. 35, 641 (2003).

[4] H. Parry, Asia Pacific Coatings J. 17, 43 (1924).

[5] H. Parry, Speciality Chemicals 23, 27 (1963).

[6] Organic Nonlinear Optical Materials (Advances in Nonlinear Optics), Ed. C. Bosshard, Springer, Berlin 2001.

[7] Molecular Nonlinear Optics, Ed. J. Zyss, Academic Press, San Diego 1994.
[8] Y. Atassi, J. Delaire, K. Nakatani, J. Phys. Chem. 99, 16325 (1995)

[9] J. Delaire, Y. Atassi, I. Maltey, K. Nakatani, Pure Appl. Opt. 5, 509 (1996).

[10] Organic Photochromic and Thermochromic Compounds, Ed. C.R. Ruglielmetti, Vol. 2, Kluwer-Academic, New York 1999, Ch. 1-2.

[11] S.P. Karna, M. Dupuis, J. Comput. Chem. 12, 237 (1991).

[12] M.W. Schmidt, K.K. Baldridge, J.A. Boatz, S.T. Elbert, M.S. Gordon, J.H. Jensen, S. Koseki, N. Matsunaga, K.A. Nguyen, S.J. Su, T.L. Windus, M. Dupuis, J.A. Montgomery, Comput. Chem. 34, 1347 (1998).

[13] B.R. Brooks, R.E. Bruccoleri, B.D. Olafson, D.J. States, S. Swaminathan, M. Karplus, J. Comput. Chem. 4, 187 (1983).

[14] F. Meyers, S.R. Marder, J.W. Perry, G. Bourhill, S. Gilmour, L.T. Cheng, B.M. Pierce, J.L. Brédas, Nonlin. Opt. 9, 59 (1995).

[15] J.F. Slater, Quantum Theory of Matter, McGraw-Hill Book Co., New York 1951, p. 391.

[16] M. Hirano, K. Osakada, H. Nohira, Miyashita, J. Org. Chem. 67, 533 (2002).

[17] J. Delaire, Y. Atassi, R. Loucif-Saibi, K. Nakatani, Nonlinear Opt. 9, 317 (1995).

[18] P.N. Prasad, D.J. Williams, Introduction to Nonlinear Optical Effect in Molecules and Polymers, Wiley, New York 1990, p. 115 